\title{
On establishing that pathology scores are quantitative
}

\author{
Donald W Drewes* \\ Department of Psychology, NC State University, USA
}

\begin{abstract}
Psychopathology researchers, like other scale developers, often fail to establish that underlying pathology scores are quantitative, concentrating instead on construction of numeric assignment procedures. By doing so, researchers risk that there may be no qualitative data relations that correspond to the quantitative structure inherent in the numerical assignment. Without representational data correspondence, numbers and their operations are devoid of meaning in a measurement context.

As a basis for quantitative establishment, a logic of quantification is presented. Pathology as a medical condition is presumed to exist in amounts referred to as magnitudes. A theory of measurable magnitudes is offered founded on seven axioms of quantity. The central conclusion from a measurement perspective is that for any ratio of two magnitudes of the same pathology, $a / b$, there exists a corresponding rational measure-number. If magnitude $b$ is a unit of measurement, then the measure-number is the number of measure units contained in magnitude $a$. Thus, measurement is definable as the act of determining the measure-number corresponding to a target magnitude $a$ given a unit of measurement $b$. The utility of the definition, however, depends upon the extent to which observable data support the quantitative hypothesis. A test that pathology scores are quantitative is provided. If supported, pathology scores can be treated as numeric in subsequent analyses.
\end{abstract}

\section{Introduction}

Two-part symptom modeling as previously presented [1] envisions a medical reality in which a pathology underlying symptom response exists in varying amounts referred to as magnitudes. Magnitudes are said to sustain the qualitative ordering relations "more than" denoted by $\succ$, "less than" denoted by $\prec$, and the equivalence relation "same as" denoted by $\sim$. In that magnitudes per se are not numeric, the arithmetic relations of $<,>$, and = are not supported. Magnitudes entertain a relation "conjoined with" denoted by $\oplus$. Pathologies whose magnitudes are hypothesized to be ordered and conjoined are said to possess a quantitative structure. No attempt was made in the cited article to establish that pathology scores are quantitative.

\section{Rise and fall of quantity considerations}

To establish pathology scores as quantitative entities requires philosophical considerations about the nature of reality-a branch of philosophy known as ontology. Historically, psychologists have eschewed philosophy claiming that empiricism is the most productive means for gathering scientific knowledge. Fechner (1887, p. 215), widely regarded as the father of quantitative psychology, responding to criticism of his psychophysical methods, charged that "all philosophical counter-demonstrations are, I think, mere writing in the sand" [2]. Resistance to philosophical criticism was most pronounced in the area of applied measurement. Following Fechner's lead, psychologists adopted his Pythagorean [3] orientation that number is the measure of all things. Some three decades later. E. L. Thorndike put forward his famous dictum that "Whatever exists at all, exists in some amount" [4]. Further implications were drawn by McCall who in 1939 proclaimed that "Anything that exists in amount can be measured" [4]. With these two ontological premises, psychologists adopted the understanding that whatever exists can be measured. The remaining unresolved issue was: What exactly is meant by measurement?

An answer was provided by S. S. Stevens in a 1946 paper on scales. According to Stevens, measurement is defined as "... the assignment of numerals to objects or events according to rule" [2]. Assignment rules are inherent in the structure of mental tests and psychological scales used as transformations to assign numbers to objects or events. Depending upon the level of admissible numerical transformations, Stevens classified scales as nominal, ordinal, linear, and ratio-a classification that remains in wide use to this day.

The existence of qualitative relations between objects or events independent of human observation, however, posed a measurement problem. Stevens circumvented this obstacle by adopting Bridgman's operationalism, a modern version of that put forth by the Chinese ideologist Mencius (BC 327-289) [4], that the meaning of a concept is drawn from the measurement operations used [2]. An object's weight, for example, is said not to have an a priori independent existence, but instead to be defined by the operation of weighing. Thus, the act of measurement is regarded as simultaneously constructing a concept as well as representing its qualitative relations by the numeric structure assigned.

Stevens' measurement by fiat was refuted by the theory of additive conjoint measurement developed by a group of mathematical psychologists [5]. Whereas Stevens' assignment rule could be any rule, additive conjoint measurement requires an assignment rule that preserves the qualitative relations observed in the data. Although touted as fundamental measurement, additive conjoint measurement failed to live up to expectations. Cliff [6] termed its failure to gain traction as "the revolution that never happened" and attributed the failure to the

*Correspondence to: Donald W Drewes, Department of Psychology, NC State University, Raleigh, NC 27695, USA, Tel: +1-919-787-3319; E-mail: donald_ drewes@ncsu.edu; conserva@mindspring.com

Key words: quantitative measurement, axioms of quantity, definition of measurement, constructive realism, microworlds, strangification

Received: February 10, 2020; Accepted: February 21, 2020; Published: February 28,2020 
complex mathematics involved, lack of demonstrated empirical power, and an inability to account for measurement error.

Consequently, Stevens' theory of measurement scales with its inherent subjectivism remains the dominant paradigm of psychology measurement. Psychologists accept the logic of equating measurement with assignment of numbers to states of a psychological attribute. However, they tend to ignore the reverse measurement implication that the attribute possesses a structure that is isomorphic in some respect to the arithmetic properties of real numbers. In the absence of this consideration, there is nothing to measure and no way of testing the claim that psychological attributes are quantitative. Michell has termed this situation a methodological thought disorder inimical to scientific discovery [2], a situation which he later referred to as pathological science [7]. Psychologists have systematically avoided the prerequisite research task of establishing that relevant variables are quantitative, concentrating instead on procedures for numeric assignment.

\section{Purpose and organization}

The intent of this commentary is to provide support logic for establishing the hypothesis that pathology scores as estimated in twopart symptom modeling are quantitative. Support logic must: (a) generalize to a domain of pathologies underlying symptom responses; (b) be founded on an explicitly stated philosophy of science; (c) generate theorems that collectively constitute a theory of measurement; (d) result in the formulation of a testable hypothesis of quantitative structure; (e) promote understanding of measurement in a medical context.

The commentary is organized according to six major sections: Rise and fall of quantity considerations; Purpose and organization; A theory of psychopathology measurement; A test for quantitative structure; Strangification-The pathway to knowledge; and a brief Integrative summary. The first section traces the changing role of quantity considerations in psychology measurement. The second states the purpose and organizational structure of the commentary. The third section describes an axiom-based theory of psychopathology measurement with subheadings devoted to the supportive ontological stance, the notion of microworlds, the transformation of the ratio of two magnitudes to a rational number, and an integrative definition of measurement. The fourth section presents a procedure for testing the quantitative hypothesis. The fifth introduces strangification as a means of knowing. A brief integrative summary is the subject of the final section.

\section{A theory of psychopathology measurement \\ An ontological stance}

The nature of the measurement theory put forth depends on resolution of the question: Do theoretical entities have an existence independent of a referencing theory? The stance as herein advanced is drawn from constructive realism, a philosophy of science propounded by F. G. Wallner [8,9], a member of the Vienna Circle. Constructive realism separates the monolithic view of reality into wirklichkeit defined as a primordial web of life in which humans are unknowingly embedded and realität defined as a nexus of constructed microworlds used as tools to control wirklichkeit. According to constructive realism, wirklichkeit exists but is unknowable. A microworld is a scientific propositional system (a model) defining and elucidating a highly specific abstract world of objects and their relations. Microworlds that facilitate wirklichkeit control are retained. Those that do not are discarded.

\section{Psychopathology microworlds}

\section{The population domain}

The psychopathology microworld of two-part modeling [1] is populated by a countable infinity of human objects. Human objects function as transducers converting the felt impact of wirklichkeit into symptom data elicited via scale instrumentation. The population is divided into two subpopulations-the first being asymptomatic of the effects of a target pathology and the second being symptomatic of the effects. Propositions regarding microworld entities and relations pertain only to the symptomatic subpopulation. The domain of the symptomatic subpopulation is between subjects, with each subject assumed to be independently and identically distributed. Individuals may vary in pathology magnitudes, but all individuals are subject to the same distributional law. It is precisely this requirement that permits sample generalization across population members. Repeated draws from a population sampling domain with these characteristics generate sample frequencies that ultimately approach the population distribution as sample size increases.

\section{Explanatory constructs}

Constructs are the key theoretical entities in a constructed microworld. Constructs are hypothesized to possess defining properties that differ in qualitative amounts across population members. These qualitative amounts are referred to as magnitudes. Individual population member magnitudes are assumed to remain stable over the duration of an observational time period. Constructs are defined to have an explanatory causal effect on observed data. In medical microworld construction, "disease" is the core construct. Each disease is said to have a single defining property referred to as a pathology serving to link the disease as an entity with a latent human condition.

\section{Axioms of quantity}

Reasoning from Thorndike's dictum, if pathological life conditions are postulated to exist, one can conclude that they must exist in some quantity or magnitude. The German mathematician O. Hölder [10] laid out seven axioms of quantity, later reinterpreted by Michell [2], as a basis for a theory of magnitudes.

I. For any two magnitudes $a$ and $b$, only one of three qualitative relations must be true. Either $a$ is the same as $b$ and $b$ is the same as a $(a \sim b, b \sim a)$; $a$ is more than $b$ and $b$ is less than $a(a \succ b, b \prec a)$; or $a$ is less than $b$ and $b$ is more than $a(a \prec b, b \succ a)$.

Axiom I allow magnitudes of a pathological life conditions to be ordered from less to more. Ties are permitted.

II. For any magnitude, there exists a different magnitude that is smaller.

Axiom II states that all pathological life conditions exist in some amount, however small. For magnitude $c$ of a pathology life condition there exists a magnitude $b$ which is less and a magnitude a that is even smaller. Thus, $\mathrm{a} \prec \mathrm{b} \prec \mathrm{c}$, from which it follows that magnitudes of a pathological life condition are continuous.

III. For any magnitudes $a, b$, and $c, a \oplus b=c$ implies that magnitudes $a$ and $b$ are the sole constituent parts of magnitude $c$.

Axiom III stipulates that separate magnitudes of a pathological life condition may be conjoined to form a larger magnitude of that condition.

IV. If $a \oplus b=c$, then $c$ is greater than $a$ and greater than $b$. 
Axiom IV claims that the magnitude of a conjoined pathological life condition is more than the magnitude of a single constituent part.

$V$. If magnitude $a$ is less than magnitude $b$, then there exists $a$ magnitude $c$ such that $a \oplus c=b$.

Axiom V proclaims that the magnitude of one pathological life condition is less than the magnitude of another if and only if the magnitude of the former life condition is a part of the latter.

VI. A magnitude that is a part of a part of another magnitude is also a part of that same magnitude; i. e. $(a \oplus b) \oplus c=a \oplus(b \oplus c)$.

Axiom VI declares that the ordering of pathological life condition magnitudes into nonoverlapping sub-parts is immaterial, indicating that the parts are independent.

VII. If the set of all magnitudes is partitioned into a lower and an upper subset so that each magnitude belongs to only a single partition, no partition is empty, and all magnitudes in the lower partition are less than any magnitude in the upper partition, then there exists a magnitude $\xi$ such that every magnitude $\xi '<\xi$ belongs in the lower partition and every magnitude $\xi " \geq \xi$ belongs to the upper partition.

Axiom VII permits all magnitudes of a pathological life condition to be divided into two ordered classes according to an existent magnitude $\xi$ serving as a cut-point.

\section{From ratio to rational number}

Using the axioms of quantity, one can show that for a magnitude $c$ and a positive integer $v$, a magnitude $a$ exists whose $v^{\text {th }}$ multiple equals $c$; i.e., $a v=c[10,87]$. Thus, $a$ is an aliquot part of $c$ in that there are $v$ independent parts, each of magnitude $a$, comprising the whole of magnitude $c$. Now, suppose that for a magnitude $d$ and a positive integer $\mu$ there exists an aliquot part $b$, where $b$ is a unit of measurement. According to Axiom I, magnitude $c$ is either $(\sim, \prec, \succ)$ magnitude $d$. If $c \sim d$, then magnitudes $c$ and $d$ are said to be commensurable and the $\sim$ relation expressed as $a v \sim b \mu$. From this equivalence, one can conclude that magnitudes $a$ and $b$ exist in the same ratio as positive integers $\mu$ and $v$; i.e., $\frac{a}{b}=\frac{\mu}{v}$. But $\frac{\mu}{v}$, besides being a ratio of two positive integers, is also interpretable as a fraction and, more important, as a positive rational number. Hölder refers to the unique rational number corresponding to the magnitude ratio $\frac{a}{b}$ as its measure-number denoted by $(a: b)[10, \mathrm{~s} 10]$. When $b$ is defined as a measure unit, then $(a: b)$ can be interpreted as the number of units of size $b$ contained in $a$.

\section{Definition of measurement}

Measurement is herein defined as:

The act of determining the measure-number corresponding to the ratio $a: b$, where $a$ is the magnitude of a specific instantiation of a target pathology and $b$ is the magnitude of a unit of measurement for the same pathology.

\section{Construct}

For the two-part symptom model [1], two contributing explanatory factors to responses from a symptomatic subpopulation are envisioned: pathological human life conditions and nonpathological human life conditions. Life condition is defined as a prolonged state of being. Drawing upon Hucklenbroich's work [11], a human life condition is said to be pathological if (a) the condition is immediately life terminating or life threatening; (b) the condition is one of prolonged pain, suffering, or discomfort; (c) the condition prevents living in social harmony according to societal norms and expectations. A human life condition is nonpathological if it is the resultant of human will and volition, imagination, illusion, delusion, or hallucination [11]. Nonpathological life conditions are treated as measurement error in two-part symptom modeling.

\section{Magnitude}

Magnitude in the context of a medical worldview is a specific instance of a pathological life condition existing at some qualitative degree of severity. Magnitudes $a$ and $b$ of the same pathological life condition can be compared accordingly to the three axiomatic qualitative relations " $a$ is more than $b$ " $(\succ)$; " $a$ is the same as $b$ " $(\sim)$; or " $b$ is less than $a$ " $(\prec)$. These three relations ensure that magnitudes can be ordered. For three magnitudes $a, b$, and $c$ of the same pathology , the axiomatic relation $(a \oplus b) \sim c$ stipulates that magnitudes $a$ and $b$ are non-overlapping parts of $c$ that can be conjoined to constitute the whole of magnitude $c$. This is a symbolic representation of the statement that the whole is equal to the sum of its parts, providing that parts are nonoverlapping.

\section{Ratio}

Ratio in comparison to number answers the question "In what proportion?" rather than "How much?" Ratios express relations between attributes and allow the relation to be scaled up or down. For example, if an ancient market had established that one ox is worth 30 fat pigs, then ratio equality determines how many pigs a buyer should expect to trade for a team of oxen. For the early Greeks, magnitudes and their ratios were distinct entities and neither considered as number. Today, the ratio of two magnitudes of the same pathological life condition is interpreted as the size of the numerator magnitude expressed in terms to the denominator magnitude considered as a unit of measurement.

\section{Measurement unit}

A measurement unit is an arbitrarily selected pathological human life condition magnitude singled out to serve as a standard. Measurement number is the number of whole and fractional measurement units contained in a target magnitude of the same pathology. The magnitude ratio $a: b$ is unaffected by a change of measurement unit, as both the ratio numerator and denominator are affected by the unit change.

\section{The act of determining}

For any two magnitudes $a$ and $b$ of the same pathology, there exists two integers $\mu$ and $v$ such that one of three relations holds. Either $a v$ $\succ b \mu, a v \sim b \mu$ or $a v \prec b \mu$. Let $c$ be a pathology magnitude targeted for measurement and $v$ an integer representing the number of constituent aliquot parts. From Holder's axioms [10], there exists a magnitude $a$ such that $a v \sim c$. Given a unit of measurement $b$ and an integer $\mu$, there co-exists a magnitude $d \sim b \mu$. The problem is to find an integer $\mu$ so that $a c \sim b \mu$, or equivalently that $c \sim d$.

The problem is procedurally dealt with by iterating $\mu$ starting with $\mu^{\prime}=1$. For a value of $\mu$, if $a v \succ b \mu$ then set $\mu^{\prime}=\mu^{\prime}+1$ and continue the iterative process. If $a v \sim b \mu$, then $\mu=\mu^{\prime}$ and discontinue iteration. If $a v$ $\prec b \mu$, then set $\mu=\mu^{\prime}-1$ and stop iterating. The result is that the measurenumber $(a: b)$ is bounded below by the rational number $\frac{\mu-1}{v}$ and above by the rational number $\frac{\mu}{v}$. The correspondence between the ratio $a: b$ and the measure-number $(a: b)$ for pathological life conditions creates a continuous positive random variable $P$ with magnitude 
ratios as the domain and positive real numbers as the range. The same procedure is used to generate a continous positive random variable $E$ for nonpathological life conditions. The random variables $P$ and $E$ are combined multiplicatively to create a random variable $Y=P \cdot E$ [1]. It is the natural $\log$ transform of the $Y$ random variable that when digitalized via scalar quantization comprises the symptom scale scores in two-part modeling [1].

\section{A test for quantitative structure}

The theorems derivable from Hölder's axioms of quantity considered in toto constitute a theory of quantitative structure. Unfortunately, there is no well-defined direct operation that allows recognition of magnitude order nor defines magnitude additive combination. Consequently, a test for quantitative structure must be derived through indirect means.

The indirect means herein proposed is the estimation of standardized latent pathology scores as defined in [1]. The conditional expectation of the latent pathology score is shown to be an unbiased estimate of the conditioned pathology score. Variance of the estimated standardized latent pathology score is shown to approach the inverse of the population maximum reliability [1]. Thus, correction of the estimated pathology scores by multiplication by the square root of the estimated maximum reliability creates a derived real random variable that approaches a standardized distribution. In that this derived real random variable is numeric, it must be quantitative [5].

Test of the quantitative hypothesis hinges on whether a single latent pathology variable can be said to underlie symptom responses, i.e. whether symptom scores are unidimensional. Unidimensional is tested by performing a single-factor CFA on the symptom correlation matrix corrected for scale coarseness [1]. If the hypothesis of unidimensionality is sustained, the data can be said to support the contention that pathology scores are quantitative. If not sustained, all that can be concluded is that the observed symptom data fail to support a quantitative hypothesis. Whether the fault lies with the data or the postulated microworld can only be resolved by subsequent research.

\section{Strangification-The pathway to knowledge}

The central premise of constructive realism is that whereas wirklichkeit is subject to human control, it cannot be understood. Control is achieved through constructing microworlds that serve as tools for mastering data [8]. Microworlds are linguistic structures of scientific propositions together with contextual rules, norms, suppositions, and customs governing their use. Microworlds coalesced by similarity into disciplines and subdisciplines are collectively referred to in constructive realism as realität [8]. But realität as the instrumentality of science cannot be equated with knowledge, as constructive realism rejects the claim that science describes the world. How then can knowledge be created and understanding achieved?

Constructive realism's answer is through a process of strangification-a translation of the linguistic and ontological framework of one microworld taken out of context and applied to another. The initial result may be the creation of a strange amalgam from the perspective of the translator, sometimes leading to interpretations regarded as absurd. A source of strangeness is that microworlds describe idealized worlds and notions that are not observable. These include concepts of infinity, number, probability, continuity, limits, population and a host of others-all sharing the common property that they cannot be directly observed. The role of the translator is to interpret these mathematical and statistical ideas in a disciplinary context.

\section{From measurement to medicine-An example of stran- gification}

The theory of measurement as herein articulated defines a microworld of propositional axioms and derived theorems supportive of the conclusion that pathology scores can be quantitatively measured. Strangification refers to the process of transference from the microworlds of measurement to the microworlds of medicine, where "disease entity" is a key theoretical concept [11]. The PTSD dimensions of Re-experiencing, Withdrawal, Arousal, and Self-Persecutions as described in [1] can be construed as pathological life conditions. Level of severity of a pathological life condition is a stand-in for the more abstract measurement concept of magnitude. Degrees can be translated as the equivalent of aliquot parts. By so doing, any severity level $c$ can be conceptually expressed as an integer number of degree multiples $v$ times the degree size $a$. If degree size $b$ is chosen as the measurement unit, then the number of degree multiples changes to $\mu$ and the ratio $\frac{a}{b}$ is approximately equal to $\frac{\mu}{v}$. But $\frac{\mu}{v}$ being a ratio of integer numbers is also a positive rational number. This metamorphism from ratio to rational number is the sine qua non for quantitative psychopathology measurement.

A cautionary note is in order. Not every data set can be expected to support the quantitative hypothesis. The reason is that the data must be unidimensional in order for standardized pathology scores to be estimated. Pathology standardized scores, if existent, are numeric and hence quantitative. Of the four PTSD pathologies reported in [1], three met the unidimensionality test and hence judged to be quantitative. The fourth, Self-Persecution, did not and was dropped from further analytic consideration.

\section{Integrative summary}

An axiom-based theory of measurement is offered founded on the ontology of constructive realism. Accordingly, reality is considered divided into twin spheres: that of wirklichkeit and realität, where wirklichkeit is an unknowable environment supportive of life and realität is a domain of multiple microworlds organized by disciplines. Microworlds are highly abstracted propositional models that guide human attempts to control wirklichkeit. Construction and testing of microworlds constitute the essence of science as high-level human action. Microworlds are tools to enhance the efficiencies and effectiveness of wirklichkeit control. But tools as means of control are not to be equated with understanding. True understanding is achieved through a process of strangification-the cross-disciplinary and crosscultural translation of a strange microworld or parts thereof into a familiar and understood disciplinary and cultural context.

In the context of constructive realism, realität is the discipline of medical disease modeling and, more specifically, the subdiscipline of psychopathology. Psychopathology is a quantitative science to the extent that the relevant pathology scores can be established to be quantitative. An axiomatic theory of quantity and a test of the quantitative hypothesis are herein offered as generic support for the prerequisite establishment task.

\section{References}

1. Drewes DW, Ho CL, Schlenger WE (2018) Symptom measurement, community sampling and the zero-problem: A case for two-part modeling. Acta Psychopathol 4: 23.

2. Michell J (1997) Quantitative science and the definition of measurement in psychology. Br J Psychol 88: 355-383. 
3. https://en.wikipedia.org/wiki/Pythagoreanism

4. $\mathrm{Xu} \mathrm{Y,} \mathrm{Li} \mathrm{H} \mathrm{(2014)} \mathrm{Existence} \mathrm{of} \mathrm{quality} \mathrm{and} \mathrm{testability} \mathrm{of} \mathrm{quantity} \mathrm{as} \mathrm{postulates} \mathrm{in} \mathrm{educational}$ and psychological measurement system. Open Cybernetics \& Systemic J 8: 884-888.

5. Borsboom D (2005) Measuring the mind: Conceptual issues in contemporary psychometrics. New York: Cambridge University Press.

6. Cliff N (1992) Abstract measurement theory and the revolution that never happened. Psychol Sci 3: 186-190.

7. Michell J (2000) Normal science, pathological science, and psychometrics. Theory and Psychol 10: 639-667.
8. Wallner FG, Jandl MJ (2006) The importance of constructive realism for the indigenous psychologies approach. In Kim U, Yang KS, Hwang KK eds., 1989. Indigenous and cultural psychology: Understanding people in context. New York: Springer Science + Business Media. pp 49-72.

9. Wallner F (1998) A New Vision of Science.

10. Michell J, Ernst C (1996) The axioms of quantity and the theory of measurement Translations from Part I of Otto Hölders's German text "Die Axiome der quantity and die lehre vom mass". J Math Psychology 40: 235-252.

11. Hucklenbroich P (2014) "Disease entity" as the key theoretical concept of medicine. $J$ Med Philos 39: 609-633.

Copyright: $(\mathbb{O} 2020$ Drewes DW. This is an open-access article distributed under the terms of the Creative Commons Attribution License, which permits unrestricted use, distribution, and reproduction in any medium, provided the original author and source are credited. 